\title{
Static hole in a two-dimensional antiferromagnet
}

\author{
D M GAITONDE, V RAMESH and S N BEHERA \\ Institute of Physics, Bhubaneswar 751005, India.
}

\begin{abstract}
The energetics of static hole in a $2 \mathrm{D}$ antiferromagnetic (AF) is studied. AF is described by magnon operators in the LSW approximation, while the holes by fermionic operators. The energy spectrum of the magnons in the presence of the hole is determined from the perturbed Green's function.
\end{abstract}

Keywords. Holes; antiferromagnet.

\section{Introduction}

Recently the problem of a static vacancy on a $2 \mathrm{D}$ AF Heisenberg square lattice has evoked a lot of interest in the context of high-temperature superconductors (Bulut et al 1989). There have been suggestions that a small amount of $\mathrm{Sr}$ in $\mathrm{La}_{2} \mathrm{CuO}_{4}$ leads to almost localized holes. Intimately related to this model are the recent experiments in which non-magnetic $\mathrm{Zn}$ has been substituted for $\mathrm{Cu}$ (Biregeneau et al 1989). The static vacancy problems is also an important limiting model for understanding the interactions of mobile holes (Kane et al 1989; Su et al 1989; Jayaprakash et al 1989) with the AF background. In our calculation we use the linear spin wave (LSW) theory for the AF background, while the static hole is described by fermionic operators unlike Bulut et al $(1989 \mathrm{a}, \mathrm{b})$ who use a purely bosonic representation for the holes as well as the host spins. Their method probably is well suited for the $\mathrm{Zn}$ impurity problem. However, it is expected that the mixed representation used by us is more suited to the problem of almost localized holes. Moreover the present formulation will also be more relevant to the mobile hole problem. In $\S 2$ the model Hamiltonian is derived. The calculation of the magnon Green function is discussed in $\$ 3$ with a summary of our results in $\S 4$.

\section{Derivation of model hamiltonian}

The Hubbard model is found to be a good starting point for the high temperature superconducting materials. In the limit of large intra-atomic repulsion $U$, and at half filling the Hubbard hamiltonian can be mapped into the Heisenberg Hamiltonian

$$
H_{0}=\frac{J}{2} \sum_{(i, j)} \mathbf{S}_{i} \cdot \mathbf{S}_{j},
$$

where the $J=4 t^{2} / U, t$ being the hopping integral. The site indices $i$ and $j$ refer to the $A($ down) and $B$ (up) sublattices respectively. The summation is carried out over all nearest neighbours. By making a cannonical transformation which rotates the spins on the $B$-sublattice by $180^{\circ}$ about the $x$-axis (Mattis 1981), $H_{0}$ can be written as

$$
H_{0}=\frac{J}{4} \sum_{i} \sum_{j(i)}\left[-S_{i}^{z} S_{j}^{z}+\frac{1}{2}\left(S_{i}^{+} S_{j}^{+}+S_{i}^{-} S_{j}^{-}\right)\right]
$$


Now $i$ runs over all lattice sites and $j$ runs over all nearest neighbours of $i$. Using the Holstein-Primakoff transformation in the LSW approximation the hamiltonian (2) can be written as

$$
H_{0}=-\frac{J N}{4}+\frac{J}{2} \sum_{k} \gamma_{k}\left(a_{k}^{\dagger} a_{-k}^{\dagger}+a_{-k} a_{k}\right)+J \sum_{k} a_{k}^{\dagger} a_{k},
$$

where $a_{k}^{\dagger}$ are magnon creation operators and $\gamma_{k}=\frac{1}{4} \sum_{\delta} \exp (i \mathbf{k} \cdot \delta)$ where $\delta$ is the nearest neighbour vector. Using the Bogoliubov transformation $H_{0}$ can be diagonalized to give

$$
H_{0}=-\frac{3 J N}{4}+\sum_{k} \omega_{k}\left(\alpha_{k}^{\dagger} \alpha_{k}+\frac{1}{2}\right)
$$

where $\omega_{k}=J\left(1-\gamma_{k}^{2}\right)^{1 / 2}$, is the magnon dispersion. The new operator $\alpha$ 's are related to the magnon operators through the transformation

$$
a_{k}=\cosh \theta_{k} \alpha_{k}+\sinh \theta_{k} \alpha_{-k}^{\dagger},
$$

where $\cosh 2 \theta_{k}=1 /\left(1-\gamma_{k}^{2}\right)^{1 / 2}$ and $\sinh 2 \theta_{k}=-\gamma_{k} /\left(1-\gamma_{k}^{2}\right)^{1 / 2}$. The removal of a down spin at a site $R_{i 0}$ or equivalently the localization of a hole at that site induces a static potential

$$
H_{\mathrm{imp}}=-\frac{J}{2} \sum_{\delta} \mathbf{S}_{i_{0}+\delta} \cdot \mathbf{S}_{i_{0}}
$$

In $H_{\text {imp }} \mathbf{S}_{i_{0}}$ which corresponds to the hole is written in terms of fermion operators and $S_{i_{0}+\delta}$ by the usual magnon operators. The total hamiltonian for the system can be written as

$$
\begin{aligned}
H= & H_{0}+H_{\text {imp }}, \\
H_{\text {imp }} & =\frac{J}{N} \sum_{k_{1} k_{2}} \gamma_{k_{1}-k_{2}} \exp \left[i\left(\mathbf{k}_{1}-\mathbf{k}_{2}\right) \cdot \mathbf{R}_{i_{0}}\right] \cosh \left(\theta_{k_{1}}+\theta_{k_{2}}\right) \alpha_{k_{2}}^{\dagger} \alpha_{k_{1}}\left(n_{i_{0} \uparrow}-n_{i_{0} \downarrow}\right) \\
& -\frac{J}{\sqrt{N}}\left\{\sum_{k_{1}} \exp \left(i \mathbf{k}_{1} \cdot \mathbf{R}_{i_{0}}\right) \gamma_{k_{1}}\left[\cosh \theta_{k_{1}} \alpha_{k_{1}}^{\dagger}+\sinh \theta_{k_{1}} \alpha_{-k_{1}}\right] c_{i_{0} \uparrow}^{\dagger} c_{i_{0} \downarrow}+\text { h.c. }\right\} .
\end{aligned}
$$

In writing (7) the terms involving $\alpha^{\dagger} \alpha^{\dagger}(\alpha \alpha)$ are dropped.

\section{Calculation of magnon Green functions}

The retarded magnon Green functions are defined as

$$
G_{k k^{\prime}}(\omega)=\left\langle\left\langle\alpha_{k} ; \alpha_{k^{\prime}}^{\dagger}\right\rangle\right\rangle_{\omega} ; \quad F_{k k^{\prime}}(\omega)=\left\langle\left\langle\alpha_{k} ; \alpha_{k^{\prime}}\right\rangle\right\rangle_{\omega^{\prime}} .
$$

The Green functions are calculated using the equation-of-motion method which gives for the free magnon Green functions (we take $J=1$ for simplicity)

$$
G_{k k^{\prime}}^{0}(\omega)=\delta_{k k^{\prime}} /\left(\omega-\omega_{k}\right) ; \quad G_{k k^{\prime}}^{0 \dagger}(\omega)=-\delta_{k k^{\prime}} /\left(\omega+\omega_{k}\right) .
$$


The average sub-lattice magnetization is defined as

$$
S_{Z}^{B}=\frac{1}{2}-\frac{1}{N} \sum_{k} \sinh ^{2} \theta_{k}
$$

The summation over momenta has been evaluated by replacing (Bulut et al 1989)

$$
\frac{1}{N} \sum_{k} \rightarrow \frac{16}{4 \pi^{2}} \int_{0}^{1} \mathrm{~d} \gamma_{k} K\left(1-\gamma_{k}^{2}\right)^{1 / 2}
$$

where $K$ is the elliptic integral of first kind. Thus

$$
\begin{aligned}
\frac{1}{N} \sum_{k} \sin h^{2} \theta_{k} & =\frac{2}{\pi^{2}} \int_{0}^{1} \mathrm{~d} \gamma_{k} K\left(1-\gamma_{k}^{2}\right)^{1 / 2}\left[1 /\left(1-\gamma_{k}^{2}\right)^{1 / 2}\right]-1, \\
& \simeq \frac{2 K(0)}{\pi^{2}} \int_{0}^{1} \mathrm{~d} \gamma_{k}\left[1 /\left(1-\gamma_{k}^{2}\right)^{1 / 2}\right]-1 .
\end{aligned}
$$

This gives the sub-lattice magnetization $S_{Z}^{B}=1 / \pi$ for the unperturbed case.

For the calculation of magnon Green functions in the presence of holes the following approximations are used:

(i) $\quad\left\langle\left(n_{i_{0} \uparrow}-n_{i_{0} \downarrow}\right) \alpha_{k} ; \alpha_{k^{\prime}}\right\rangle_{\omega} \simeq M \ll \alpha_{k} ; \alpha_{k^{\prime}} \rrbracket_{\omega}$,

where $M=\left\langle n_{i_{0} \uparrow}-n_{i_{0} \downarrow}\right\rangle \approx 2 S_{z}^{B}$

(ii) The two-particle Green function $\left\langle\left\langle c_{i_{0} \sigma}^{\dagger} c_{i_{0}-\sigma} ; c_{i_{0} \sigma^{\prime}}^{\dagger} c_{i_{0}-\sigma^{\prime}}\right\rangle_{\omega}\right.$ is evaluated only in the Ising limit.

(iii) To solve the resulting integral equation we assume the separability of $\gamma_{k_{1}-k_{2}} \approx \gamma_{k_{1}} \gamma_{k_{2}}$ following Bulut et al (1989).

Furthermore on defining the magnon Green function in a matrix form and carrying out the configurational averaging over the hole sites the equation can be reduced to the Dyson form which when solved explicitly gives for the Green functions

$$
\begin{aligned}
G(k, \omega) & =\frac{\omega+\omega_{k}+\Sigma^{+-}(k, \omega)}{\left(\omega-\omega_{k}-\Sigma^{-+}(k, \omega)\right)\left(\omega+\omega_{k}+\Sigma^{+-}(k, \omega)\right)+\Sigma^{++}(k, \omega) \Sigma^{--}(k, \omega)}, \\
F(k, \omega) & =\frac{-\Sigma^{--}(k, \omega)}{\left(\omega-\omega_{k}-\Sigma^{-+}(k, \omega)\right)\left(\omega+\omega_{k}+\Sigma^{+-}(k, \omega)\right)+\Sigma^{++}(k, \omega) \Sigma^{--}(k, \omega)}
\end{aligned}
$$

Similar results for the $G^{\dagger}$ and $F^{+}$follows from (15) by replacing $\omega \rightarrow-\omega$. The self-energies are given by

$$
\begin{aligned}
& \Sigma^{+-}(k, \omega)= \\
& M \delta \gamma_{k}^{2}\left[\frac{\cosh ^{2} \theta_{k}}{f_{2}(\omega)}\left(1-\frac{1}{(\omega+M) f_{2}(\omega)}\right)+\frac{\sinh ^{2} \theta_{k}}{f_{1}(\omega)}\left(1+\frac{1}{(\omega-M) f_{1}(\omega)}\right)\right] \\
& \Sigma^{--}(k, \omega)=M \delta \gamma_{k}^{2} \sinh 2 \theta_{k}\left[\frac{1}{(\omega-M) f_{1}(\omega) f_{2}(-\omega)}-\frac{1}{(\omega+M) f_{1}(-\omega) f_{2}(\omega)}\right]
\end{aligned}
$$


where $\delta=$ the dopant concentration

$$
\begin{aligned}
f_{1}(\omega) & =1-M(1+\omega) \frac{h(\omega)}{\pi}-\frac{M}{4} \\
f_{2}(\omega) & =1-M(1-\omega) \frac{h(\omega)}{\pi}+\frac{M}{4} \\
h(\omega) & =1-\frac{\pi}{2} \omega-2\left(\omega^{2}-1\right)^{1 / 2} \arctan \left(\left(\frac{\omega-1}{\omega+1}\right)^{1 / 2}\right) \quad \text { for }|\omega|>1 \\
& =1-\frac{\pi}{2} \omega+\left(1-\omega^{2}\right)^{1 / 2} \ln \left|\frac{\left(1-\omega^{2}\right)^{1 / 2}-\omega-1}{\left(1-\omega^{2}\right)^{1 / 2}+\omega-1}\right| \quad \text { for }|\omega|<1
\end{aligned}
$$

\section{Results and discussion}

The self-energies depend linearly on $\delta$. For low dopant concentration, dropping the term proportional to $\delta^{2}$, the Green functions become

$$
\begin{aligned}
& G(k, \omega)=\frac{1}{\omega-\omega_{k}-\Sigma^{-+}(k, \omega)}, \\
& F(k, \omega)=\frac{-\Sigma^{--}(k, \omega)}{\left(\omega-\omega_{k}-\Sigma^{-+}(k, \omega)\right)\left(\omega+\omega_{k}+\Sigma^{+-}(k, \omega)\right)} .
\end{aligned}
$$

The poles of the Green functions have been studied numerically. The modified magnon frequency will be the solution of the equation $\omega-\omega_{k}-\Sigma^{-+}(k, \omega)=0$, which for the low dopant concentration does not have any solution corresponding to a localized mode. The modification of magnon frequency is negligible up to concentration $\delta=0.002$. The magnon band formation breaks down as soon as the impurity concentration goes above this value. This is probably due to the breakdown of the LSW approximation.

The localized modes due to the presence of the holes appear as the poles of the self-energy term $\Sigma^{--}(k, \omega)$. The results show two localized modes with frequencies at 2.22 and 1.61 , with the magnon band lying between 0 and 1 . The localized mode at $\omega=M$ may be neglected since this is a result of the Ising limit approximation which was used to calculate the two-particle Green functions. The hole Green function has also been calculated by us, whose result will be discussed elsewhere.

\section{References}

Birgeneau R J et al 1989 Z. Phys. (to be published)

Bulut N et al 1989a Phys. Rev. Lett. 622192

Bulut $\mathrm{N}$ et al $1989 \mathrm{~b}$ ITP Preprint

Jayaprakash C et al 1989 Phys. Rev. B40 2160

Kane C L et al 1989 Phys. Rev. B39 6880

Mattis D C 1981 in The theory of magnetism I (Springer Verlag)

Su Z B et al 1989 Phys. Rev. Lett. 631318 